\section{Case Reports in Dermatology}

\title{
A Case Report of Cushing's Disease Presenting as Hair Loss
}

\author{
Emily G. Lefkowitz ${ }^{a} \quad$ Jack P. Cossman ${ }^{b} \quad$ John B. Fournier ${ }^{b-d}$ \\ a Boston University School of Medicine, Boston, MA, USA; ${ }^{b}$ Department of Dermatology, \\ Roger Williams Medical Center, Providence, RI, USA; ${ }^{\circ}$ Department of Dermatology, Boston \\ University School of Medicine, Boston, MA, USA; ${ }^{d}$ Inpatient Dermatology Consultative \\ Service, Roger Williams Medical Center, Providence, RI, USA
}

\section{Keywords}

Cushing's disease $\cdot$ Female-pattern hair loss · Androgenetic alopecia

\begin{abstract}
Cushing's syndrome is a rare endocrine disorder that comprises a large group of signs and symptoms resulting from chronic exposure to excess corticosteroids. Most cases of Cushing's syndrome are due to increased adrenocorticotropic hormone production from a pituitary adenoma, which is referred to as Cushing's disease. Most of the signs and symptoms are nonspecific and common in the general population, making a diagnosis often challenging. However, several dermatological manifestations, such as fragile skin, easy bruising, and reddish purple striae, are more discriminatory. Because uncontrolled Cushing's syndrome of any etiology is associated with substantial morbidity, including increased cardiovascular disease and mortality, it is important to make an early diagnosis. Unfortunately, median delays of 2 years to diagnosis have been reported. We report a case of a woman who had multiple dermatological findings, including facial plethora, easy bruising, violaceous striae, hirsutism, and acne, the latter 2 signs reflecting androgen excess. Of interest, our patient presented with a chief complaint of hair loss, a common complaint in the general population that occurs with a greater frequency in patients with Cushing's disease and is attributed to androgenetic alopecia, but it is rarely the presenting symptom.




\section{Introduction}

Cushing's syndrome is characterized by signs and symptoms that result from prolonged exposure to excessive plasma corticosteroids. While the most common cause is iatrogenic from medically prescribed corticosteroids, endogenous Cushing's syndrome is a relatively rare disease. A recent US-based study estimated the incidence of endogenous Cushing's syndrome at 8 per million people [1]. Pituitary-secreting tumors, first described by Harvey Cushing in 1912 and now referred to as Cushing's disease, account for approximately 70\% of cases, while ectopic adrenocorticotropic hormone (ACTH)-secreting tumors and adrenal tumors account for 10 and $20 \%$ of cases, respectively [2].

Although the diagnosis of Cushing's syndrome can be straightforward, when several clinical findings are present, it is often challenging to make the diagnosis. None of the symptoms or signs are pathognomonic of the syndrome, and many symptoms (such as obesity, hypertension, glucose intolerance, weight gain, fatigue, weakness, menstrual abnormalities, and depression) are found in the general population. In contrast, the dermatological manifestations of Cushing's syndrome that include skin atrophy, alopecia, easy bruisability, and striae are less commonly observed in other individuals [3]. We report an unusual case of a woman who presented with a chief complaint of hair loss and who was found to have Cushing's disease.

\section{Case Report}

A 33-year-old female with a history of gastritis, depression, and obesity presented to the dermatology clinic with a chief complaint of hair loss. She reported that the hair on her head began to thin rapidly 6 months prior to presentation, although she had been experiencing progressive thinning for about 1 year. There was minimal improvement with daily minoxidil $5 \%$ foam after 3 months.

The patient had a pituitary microadenoma that was known to exist for at least a decade, but a recent magnetic resonance imaging had shown the microadenoma to be stable in size. Serum prolactin, follicle-stimulating hormone, and luteinizing hormone levels were normal.

Physical examination showed a middle-aged female in no acute distress with facial plethora and prominent truncal, facial, and nuchal adiposity. Examination of the skin revealed diffuse nonscarring alopecia on the frontal scalp and vertex of the scalp (Fig. 1), and miniaturized hairs were seen on dermoscopy. To a lesser extent, the density of temporal and occipital hair was also decreased. There were erythematous inflammatory papules and pustules on her face and mid to lower back, violaceous striae on the abdomen and axillae, and ecchymoses on the abdomen and extremities. Facial hirsutism and patches of coarse hypertrichosis on her back were also noted (Fig. 2).

Serum ferritin, iron, thyroid-stimulating hormone, antinuclear antibody, albumin, total protein, and vitamin $B_{12}$ were all negative or within normal limits. Punch biopsy from the scalp showed a normal number of follicles without fibrosis or follicular dropout but a striking increase in the ratio of catagen and telogen hairs to anagen hairs and a sparse peribulbar lymphocytic infiltrate.

Subsequent tests were ordered: 24 -h urine cortisol: $384 \mu \mathrm{g}$ (range 3.5-45 $\mu \mathrm{g} / 24 \mathrm{~h}$ ), dehydroepiandrosterone sulfate: $380 \mu \mathrm{g} / \mathrm{dL}$ (range $45-270 \mu \mathrm{g} / \mathrm{dL}$ ), and ACTH: $76 \mathrm{pg} / \mathrm{mL}$ (range $9-52 \mathrm{pg} / \mathrm{mL}$ ). The results were consistent with a diagnosis of Cushing's disease. The 
patient was referred to an endocrinologist and subsequently underwent a transsphenoidal resection of the pituitary microadenoma.

\section{Discussion}

Dermatologists may encounter skin findings that reflect an underlying endocrine disorder. While many of the signs and symptoms of Cushing's syndrome are nonspecific, those features that best distinguish Cushing's syndrome are proximal muscle weakness, facial plethora, easy bruising, and purple (violaceous) striae [3]; the latter 3 dermatological findings were seen in our patient on physical examination.

The often prominent skin findings reflect the hypercatabolic effects of hypercortisolism - inhibition of epidermal cell division and collagen synthesis, resulting in thinning of the stratum corneum and loss of subcutaneous fat [4]. Skin atrophy may be prominent, and the loss of subcutaneous connective tissue results in easy bruising after minimal injury. The atrophy and disruption of collagenous subcutaneous fibers lead to the development of broad, purple striae because the increasingly thin skin does not hide the color of venous blood in the underlying dermis. Another skin finding is hyperpigmentation due to excess $\mathrm{ACTH}$, which is most commonly seen in ectopic ACTH syndrome, less commonly in Cushing's disease (i.e., pituitary-secreting ACTH tumor), and never in adrenal Cushing's syndrome; the hyperpigmentation is a result of ACTH binding to melanocyte-stimulating hormone receptors.

In contrast to the aforementioned skin changes, female baldness or female hair loss has been variably reported and is rarely the chief complaint of a patient presenting with Cushing's syndrome [2]. In a review of Cushing's syndrome that included 7 case series of 33-100 patients, 4 case series did not report female baldness, whereas it was reported in 3 of the case series with an incidence ranging from 13 to 51\% [5]. More recently, the European Registry on Cushing's Syndrome reported "hair loss" in 110 of 351 (31\%) patients with Cushing's syndrome and in 76 of 224 (34\%) patients in the subset with Cushing's disease [6]. In a recent matched case-control study using a commercial health-care insurance claims database that included 1,875 patients with Cushing's syndrome, female balding was found to be 5 times more common than in the matched controls; the combination of weakness/fatigue and female baldness was 10 times more common [7].

Female balding in Cushing's syndrome results from androgen hypersecretion that occurs in ACTH-dependent forms (i.e., pituitary or ectopic ACTH tumors) or with adrenocortical cancers but never with adrenal adenomas. Androgenetic alopecia, often referred to as female-pattern hair loss (FPHL), is a nonscarring form of hair loss in which the growth (anagen) phase of hair follicles is shortened resulting in follicular miniaturization [8]. The frontal scalp and vertex of the scalp are the primary sites of involvement, and the occipital scalp is usually relatively spared. The exact pattern of hair loss varies among women but typically presents with a diffuse central thinning pattern or with prominent frontal scalp thinning referred to as a "Christmas tree" pattern.

FPHL is a common condition with a reported prevalence of $19 \%$ in 1 series of 1,006 Caucasian women in the United States [9]. While most women show no signs of androgen excess, a careful workup is warranted. In a series of 109 women referred for the evaluation of moderate to severe FPHL, laboratory evidence for hyperandrogenism was present in 39\%, with the most common diagnosis being polycystic ovarian syndrome [10]. Our patient was noted to have an elevated dehydroepiandrosterone sulfate level, a metabolic intermediate in 
the biosynthesis of androgens. Women who present with FPHL should be evaluated for associated signs or symptoms of androgen excess, such as hirsutism, moderate to severe acne, and irregular menses or amenorrhea. As expected, our patient who presented with alopecia had associated findings of hirsutism and acne. It should be noted, however, that in Cushing's syndrome the amenorrhea is likely due to cortisol-mediated inhibition of gonadotrophin release rather than to hyperandrogenism.

In a review of Cushing's syndrome, Findling and Raff [11] noted that the diagnosis of Cushing's syndrome "is the most challenging problem in clinical endocrinology." Patients with Cushing's syndrome and persistent hypercortisolism have a 4-5 times excess mortality compared to the general population, highlighting the urgency of diagnosis [12]. In the European Registry on Cushing's Syndrome, the median delay to diagnosis was a remarkable 2 years [6]. They found that general practitioners were consulted $76 \%$ of the time, endocrinologists $25 \%$, gynecologists $24 \%$, psychiatrists/psychologists $12 \%$, rheumatologists $11 \%$, and dermatologists $8 \%$ of the time.

As noted above, the majority of clinical signs and symptoms of Cushing's syndrome are relatively nonspecific, whereas dermatological manifestations, such as fragile skin, easy bruising, and purple striae, are more discriminatory [3]. Broder et al. [7] noted hirsutism to be 61 times more common in Cushing's syndrome than in the general population. In contrast, FPHL is commonly seen in the general population, although with a 5 times greater frequency in patients with Cushing's syndrome.

Our patient presented with a history of a pituitary adenoma and findings of central obesity, easy bruising, purple striae, hypertrichosis, and female balding, and a diagnosis was rapidly made. Of interest, the case was unusual in that it was the hair loss that caused the patient to seek medical attention.

\section{Statement of Ethics}

The authors have no ethical conflicts to disclose. The patient has given her informed consent, including the use of the photographs.

\section{Disclosure Statement}

The authors have no conflicts of interest to disclose. No funding support was obtained for this work.

\section{References}

1 Broder MS, Neary MP, Chang E, et al: Incidence of Cushing's syndrome and Cushing's disease in commercially-insured patients $<65$ years old in the United States. Pituitary 2015;18:283-289.

2 Newell-Price J, Bertagna X, Grossman AB, Nieman LK: Cushing's syndrome. Lancet 2006;367:16051617.

-3 Nieman LK, Biller BMK, Findling JW, et al: The diagnosis of Cushing's syndrome: an Endocrine Society Clinical Practice Guideline. J Clin Endocrinol Metab 2008;93:1526-1540.

$\checkmark 4$ Jabbour SA: Cutaneous manifestations of endocrine disorders: a guide for dermatologists. Am J Clin Dermatol 2003;4:315-331.

5 Newell-Price J, Trainer P, Bessler M, Grossman A: The diagnosis and differential diagnosis of Cushing's syndrome and pseudo-Cushing's states. Endocr Rev 1998;19:647-672. 


\section{Case Reports in Dermatology}

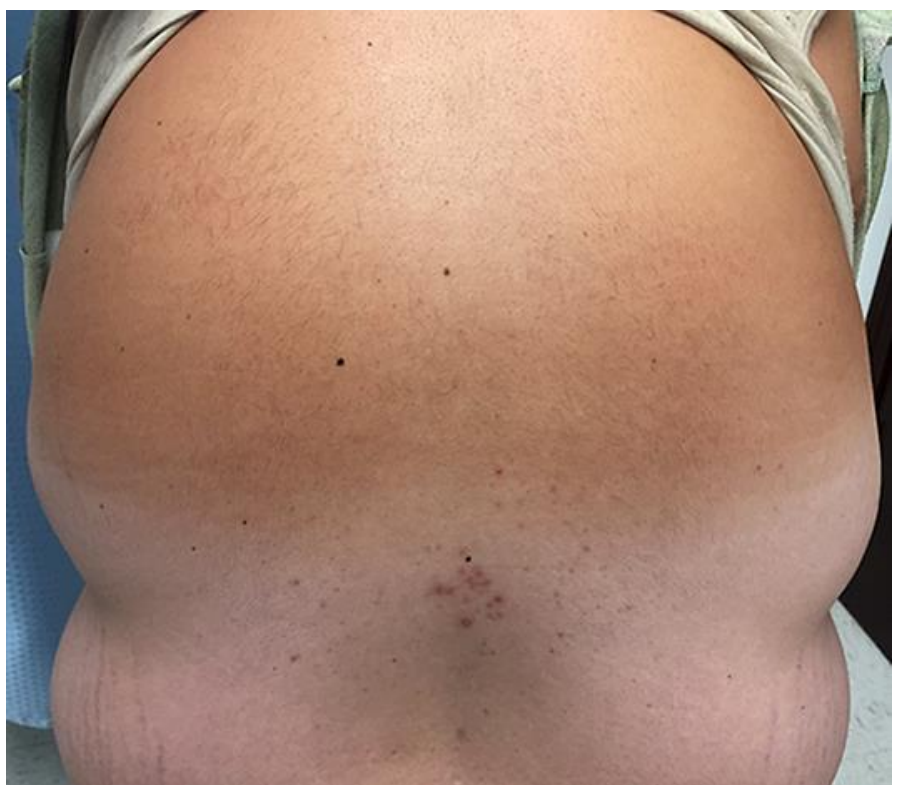

Fig. 2. Coarse patches of hypertrichosis and erythematous inflammatory papules and pustules on the mid to lower back and violaceous striae on the flanks bilaterally. 